5 International Chamber of Commerce, European Society for Opinion and Marketing Research. ICC/ESOMAR International Code On Market and Social Research www.esomar.org/index. php/codes-guidelines.html Date last updated: December 2007.

6 Heck JE, Stücker I, Allwright S, et al. Home and workplace smoking bans in Italy, Ireland, Sweden, France and the Czech Republic. Eur Respir J 2010; 35: 969-979.

7 Winickoff JP, Gottlieb M, Mello MM. Regulation of smoking in public housing. New Eng J Med 2010; 362: 2319-2325.

8 Dept of Housing and Urban Development, Office of Public and Indian Housing. Non-Smoking Policies in Public Housing
(Memorandum). www.hud.gov/offices/pih/publications/notices/ 09/pih2009-21.pdf Date last accessed: June 27, 2010. Date last updated: July 17, 2009.

9 Tønnesen P, Carrozzi L, Fagerström KO, et al. Smoking cessation in patients with respiratory diseases: a high priority, integral component of therapy. Eur Respir J 2007; 29: 390-417.

10 Sigsgaard T, Clancy L, Forastiere F, et al. 20 years of research and advocacy for a healthy and tobacco-free environment. Eur Respir $J$ 2010; 36: 1-3.

\title{
Shorter treatment duration for selected patients with multidrug-resistant tuberculosis
}

\section{To the Editors:}

Tuberculosis (TB) is still an infectious disease of public health importance today [1]. Of particular concern is the occurrence of multidrug-resistant (MDR)-TB with resistance to at least isoniazid and rifampicin, and extensively drug-resistant (XDR)-TB with additional resistance to any fluoroquinolone and at least one of the second-line injectable drugs, including kanamycin, amikacin and capreomycin. MDR- and XDR-TB need prolonged treatment duration, from 18 to 24 months after sputum culture conversion, as recommended by the World Health Organization (WHO) [2]. A prolonged duration of treatment may lead to poor adherence, higher cost and undue toxicity. In our previous studies, we found that the use of ofloxacin/levofloxacin-containing regimens had a favourable effect on MDR-TB outcome [3, 4]. Our studies also suggested that shorter treatment duration (mean duration 14 months) might be feasible in selected MDR-TB patients. Based on this finding, the treatment duration of selected MDR-TB patients under programmatic management in Hong Kong was changed from the rigid enforcement of $\geqslant 18$ months after sputum conversion to an individualised duration tailored by the physician's judgement. The determinants favouring longer treatment included extensive drug resistance, delayed sputum culture conversion, greater extent of radiographic disease (including cavities), presence of diabetes or silicosis, and extrapulmonary disease. Out of these factors, the extent of drug resistance was the most important. Ofloxacin-resistant MDRTB was arbitrarily given a prolonged treatment based on our previous studies $[3,4]$. Despite a relatively high TB incidence (76 cases per 100,000 population in 2009), both TB and MDR$\mathrm{TB}$ rates are actually decreasing under the local TB control programme [5]. Therefore, we conducted a retrospective evaluation of the long-term outcomes of our MDR-TB patients, focusing on the factors affecting the efficacy and relapse rate of a shorter treatment duration.

All consecutive MDR-TB cases under the programmatic management of the Hong Kong Tuberculosis and Chest Service from 1997 to 2006 were included in this retrospective cohort. Case categories and clinical outcomes at 2 yrs were classified according to the criteria proposed by LASERSON et al. [6]. The final clinical status of successful patients (cured or having completed treatment) and the survival status of the entire cohort were ascertained until December 31, 2009. Patients lost to follow-up were tracked through the territorywide TB notification registry and death registry, which captured TB cases and deaths, respectively, all over Hong Kong. For analysis of survival and relapse, incidence densities were compared between different categories in univariate analysis, followed by Cox proportional hazard analysis in multivariate analysis. Statistical analyses were performed with EPI Info version 6.04 (Centers for Diseases Control and Prevention, Atlanta, GA, USA) and SPSS version 16 (IBM, Quarry Bay, Hong Kong). A two-tailed p-value $<0.05$ was taken as statistically significant.

The study was approved by the Ethics Committee of the Department of Health, Hong Kong. All patients were informed at the time of care delivery that the clinical data collected would be used for statistical analysis and research purposes.

There were 270 MDR-TB patients (including 27 XDR-TB patients) in the cohort. They included $245(90.7 \%)$ patients with pulmonary TB only, six (2.2\%) patients with extrapulmonary TB only, and 19 (7.1\%) patients with both pulmonary and extrapulmonary involvement. Voluntary HIV testing was offered to all patients, with 45 declining the test. Three $(1.2 \%)$ out of 235 patients tested were HIV-positive and they were all non-XDR, MDR-TB patients.

Of all included subjects, $187(69.3 \%)$ patients were cured or completed treatment, $26(9.6 \%)$ died during treatment, $45(16.7 \%)$ defaulted on treatment (with median treatment duration 5 months, range $0.5-18$ months), nine (3.3\%) were transferred out and three $(1.1 \%)$ had treatment failure. All failure patients had XDR-TB. Lung resections (eight lobectomies, one lobectomy plus segmentectomy, and one pneumonectomy) were performed in a total of 10 patients (three XDR-TB and seven non-XDR, MDR-TB).

Among patients successfully treated, significantly longer duration of treatment was associated with XDR-TB compared with other MDR-TB patients (median duration 21 versus 15 months; $\mathrm{p}<0.01$ ). $55.1 \%$ (97 out of 176 ) of the successfully treated non-XDR, MDR-TB patients were treated for $\leqslant 15$ months (median 13.5 months, range 11-15 months). 


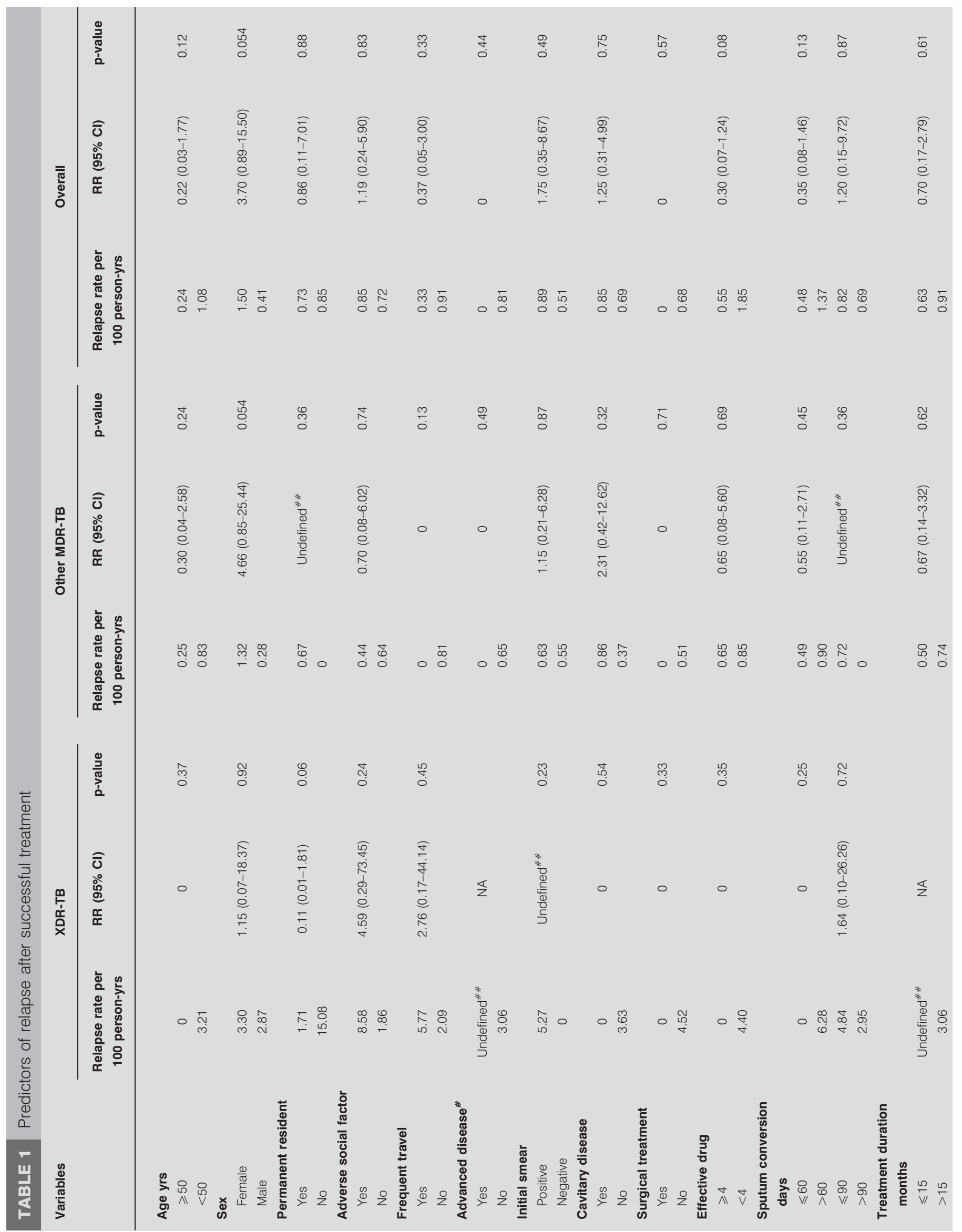




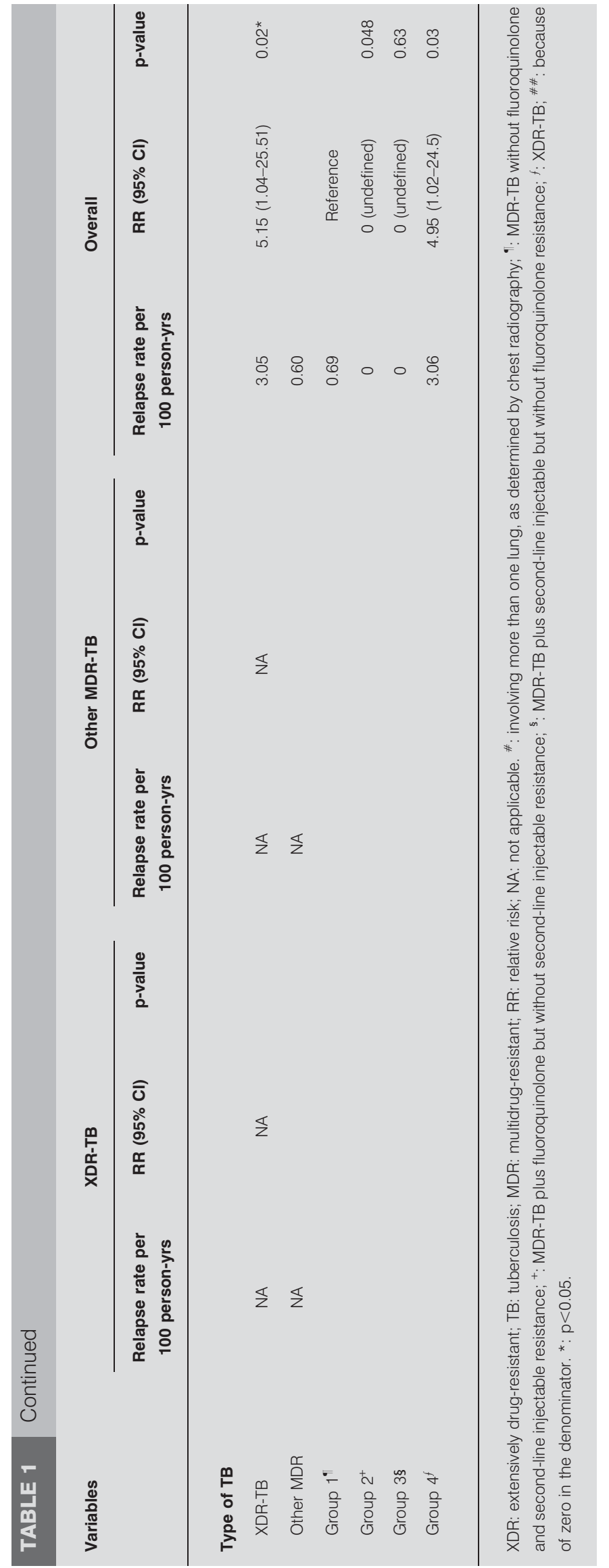

Noncavitary disease at baseline $(\mathrm{p}=0.048)$, and sputum culture conversion at the second $(p=0.009)$ and third $(p=0.013)$ months after initiation of MDR-TB treatment were associated with the use of a shorter duration ( $\leqslant 15$ months) of treatment in these non-XDR patients. There were eight $(4.3 \%)$ relapses after a mean $\pm S D$ follow-up duration of $5.7 \pm 3.1 \mathrm{yrs}$ after the end of second-line treatment, with a relapse rate of 0.75 per 100 person-yrs. Restriction fragment length polymorphism analysis was not performed, but the Mycobacterium tuberculosis isolates in all six (two XDR- and four MDR-TB) cases confirmed bacteriological relapses with identical bacillary susceptibility patterns as the original strains. In the remaining two MDR-TB relapses, one was confirmed histologically as pulmonary TB and TB colitis. The other was a radiological relapse 2 yrs after treatment completion with improvement of chest radiography after re-treatment. In the subgroup analysis based on the susceptibility pattern stratifying $M$. tuberculosis strains into four groups (table 1), MDR strains with additional resistance to either fluoroquinolone or second-line injectables (but not both) did not appear to have a higher relapse rate. All except two of these patients (both with additional resistance to second-line injectables alone) were given $\geqslant 18$ months of treatment under our programmatic setting. XDR-TB was the only factor that predicted relapse in the cohort, and none of the other factors showed an independent effect after stratification by XDR-TB status (table 1). Among successfully treated simple MDR-TB patients (group 1), no difference ( 0.50 versus 0.74 per 100 person-yrs; $p=0.62)$ in the relapse rate was found between those who had $\leqslant 15$ months of treatment $(\sim 12$ months after culture conversion) and those who had longer treatment.

There were 70 known deaths (26 during treatment and 44 after treatment completion) after a mean \pm SD follow-up duration of $6.7 \pm 3.4$ yrs: $12(44.4 \%)$ in XDR-TB and $58(23.9 \%)$ in other MDR-TB patients $(\mathrm{p}=0.021)$. TB-related death was found in eight $(29.6 \%)$ XDR-TB patients compared with eight $(3.3 \%)$ in other MDR-TB patients $(p=0.001)$. Among the non-XDR, MDRTB patients successfully treated, the mortality rate was $14.4 \%$ (14 patients) after treatment completion in those who received $\leqslant 15$ months of treatment versus $25.3 \%$ (20 patients) in those received $>15$ months of treatment (Chi-squared $p=0.07$ ).

Relapse of TB has been attributed to the failure to eradicate "persisters", which are characterised by having brief spurts of metabolic activity punctuating with periods of dormancy, in contrast to treatment failure attributable to the inability to kill rapidly dividing bacteria [7]. The relapse rate $(4.3 \%$ with mean follow-up of 5.7 yrs) observed among successfully treated MDRTB patients in the present cohort was considerably higher than the overall relapse rates among TB patients in Hong Kong $(0.9 \%$ in $2.5 \mathrm{yrs}$ ) [8], but lower than in a MDR-TB series in Peru (5.2\% in 2 yrs) [9]. The higher relapse rate among patients with XDR-TB (group 4 in the subgroup analysis) may have been due to the loss of the most potent anti-TB agents: rifampicin and the two pivotal groups of second-line drugs (fluoroquinolone and the injectables) in their treatment regimens, despite having prolonged treatment duration of at least 18 months after sputum conversion. The excess relapse rate for $\mathrm{XDR}-\mathrm{TB}$ underscores the importance of key XDR-TB-defining drugs in the treatment regimen, as previously demonstrated by other studies [10, 11]. The duration of treatment for MDR-TB currently recommended by WHO is based on expert opinion and not on evidence from 
randomised controlled trials. Among successfully treated nonXDR, MDR-TB patients in the present study, 55.1\% were treated for $\leqslant 15$ months. Therefore, for simple MDR-TB patients on levofloxacin-based regimens, a total of $\sim 15$ months of therapy (or $1 \mathrm{yr}$ after sputum culture conversion, as the conversion generally takes 1-3 months among those with favourable response to treatment) might be sufficient, instead of 1824 months [3, 4]. Treatment duration of $\geqslant 18$ months after culture conversion is probably necessary for XDR-TB and MDR-TB with fluoroquinlone or second-line injectable resistance. The low relapse after nearly $5 \mathrm{yrs}$ of follow-up posttreatment further confirmed the efficacy of the shorter regimen, which is likely to improve adherence, save valuable resources (manpower and expense) and reduce adverse effects. In our previous study [3] and in another study on MDR-TB [12], sputum conversion at an early stage was a predictor of treatment success. Early culture conversion might, therefore, serve as an indicator for use of a shorter regimen among non-XDR, MDR-TB patients without extensive and/or cavitary disease. In this regard, a new category, "short-term treatment completion", has also been used in Korean studies [13] to include those patients who had $>6$ but $<18$ months of treatment, with more than three consecutive negative cultures and good clinical response. In those studies, the relapse rate in this category $(12.6 \%)$, although higher than the overall relapse rate $(8.9 \%)$, was not statistically significant. Another cohort study among MDR-TB patients naïve to second-line anti-TB drugs also showed that by using highdose gatifloxacin and isoniazid plus other active secondline drugs, treatment duration could be shortened to 9-12 months [14].

The present longitudinal analysis of a MDR-TB cohort under treatment in a programmatic setting has limitations inherent to the study design. The choice of drug regimens and their durations could have been confounded by differences in baseline characteristics and treatment response. However, with the intrinsic difficulties associated with randomised controlled trials on the treatment of MDR-TB, the "optimal" duration of treatment for MDR-TB has been addressed only by longitudinal follow-up of treatment cohorts [3, 14]. The availability of high-quality laboratory support (a WHOdesignated supranational TB reference laboratory) and individualised treatment through good health infrastructure to provide reliable management of drug-resistant $\mathrm{TB}$, constitutes the main strength of this study. In contrast with similar studies $[9,13]$, long-term follow-up data (mean duration of $5.7 \mathrm{yrs}$ after cessation of treatment) were available. Besides regular follow-up of patients, defaulters were also tracked through the territory-wide $\mathrm{TB}$ notification registry and death registry to ensure more complete ascertainment of the relevant study endpoints. It is therefore hoped that our findings will help to inform the treatment of MDR-TB before results from carefully designed clinical trials are available to address the optimal treatment strategies more definitively [15].

\footnotetext{
E.C.C. Leung*, W.W. Yew ${ }^{\#}$, C.C. Leung*, W.M. Leung* and C.M. Tam*
}

*Tuberculosis and Chest Service, Centre for Health Protection, Dept of Health, Wan Chai, and "Tuberculosis and Chest Unit, Grantham Hospital, Wong Chuk Hang, Aberdeen, Hong Kong.

Correspondence: E.C.C. Leung, Wan Chai Chest Clinic, 99 Kennedy Road, Wan Chai, Hong Kong. E-mail: eric_leung@ dh.gov.hk

Statement of Interest: A statement of interest for W.W. Yew can be found at www.erj.ersjournals.com/site/misc/statements.xhtml

\section{REFERENCES}

1 World Health Organization. Global Tuberculosis Control - a Short Update to the 2009 Report. WHO/HTM/TB/2009.426. Geneva, WHO, 2009.

2 World Health Organization. Guidelines for the Programmatic Management of Drug-Resistant Tuberculosis. Emergency Update 2008. WHO/HTM/ TB/2008.402:1-247. Geneva, WHO, 2008.

3 Yew WW, Chan CK, Leung CC, et al. Outcomes of patients with multidrug-resistant pulmonary tuberculosis treated with ofloxacin/levofloxacin-containing regimens. Chest 2000; 117: 744-751.

4 Yew WW, Chan $\mathrm{CK}$, Chau $\mathrm{CH}$, et al. Comparative roles of levofloxacin and ofloxacin in the treatment of multidrug-resistant tuberculosis: preliminary results of a retrospective study from Hong Kong. Chest 2003; 124: 1476-1481.

5 World Health Organization. Anti-Tuberculosis Drug Resistance in the World, 4th Global Report. Geneva, WHO, 2008.

6 Laserson KF, Thorpe LE, Leimane V, et al. Speaking the same language: treatment outcome definitions for multidrug-resistant tuberculosis. Int J Tuberc Lung Dis 2005; 9: 640-645.

7 Fox W, Ellard GA, Mitchison DA. Studies on the treatment of tuberculosis undertaken by the British Medical Research Council tuberculosis units, 1946-1986, with relevant subsequent publications. Int J Tuberc Lung Dis 1999; 3: S231-S279.

8 Chang KC, Leung CC, Yew WW, et al. A nested case control study on treatment-related risk factors for early relapse of tuberculosis. Am J Respir Crit Care Med 2004; 170: 1124-1130.

9 Becerra MC, Appleton SC, Franke MF, et al. Recurrence after treatment for pulmonary multidrug-resistant tuberculosis. Clin Infect Dis 2010; 51: 709-711.

10 Migliori GB, Lange C, Girardi E, et al. Fluoroquinolones: are they essential to treat multidrug-resistant tuberculosis? Eur Respir J 2008; 31: 904-905.

11 Chan ED, Strand MJ, Iseman MD. Multidrug-resistant tuberculosis (TB) resistant to fluoroquinolones and streptomycin but susceptible to second-line injection therapy has a better prognosis than extensively drug-resistant TB. Clin Infect Dis 2009; 48: e50-e52.

12 Leimane V, Dravniece G, Riekstina V, et al. Treatment outcome of multidrug/extensively drug-resistant tuberculosis in Latvia, 20002004. Eur Respir J 2010; 36: 584-593.

13 Kim DH, Kim HJ, Park SK, et al. Treatment outcomes and longterm survival in patients with extensively drug-resistant tuberculosis. Am J Respir Crit Care Med 2008; 178: 1075-1082.

14 Van Deun A, Maug AK, Salim MA, et al. Short, highly effective and inexpensive standardized treatment of multidrug-resistant tuberculosis. Am J Respir Crit Care Med 2010; 182: 684-692.

15 Cobelens FG, Heldal E, Kimerling ME, et al. Scaling up programmatic management of drug-resistant tuberculosis: a prioritized research agenda. PLoS Med 2008; 5: e150.

DOI: $10.1183 / 09031936.00186310$ 